\title{
Identification and Quantitation of Cashmere (Pashmina) Fiber and Wool Using Novel Microchip Based Real-Time PCR Technology
}

\author{
Rajwant Gill, Sikander Gill, Maxim Slyadnev, Alexander Stroganov \\ Lumex Instruments Canada, Mission, Canada \\ Email: sikander.gill@lumexinstruments.com
}

How to cite this paper: Gill, R., Gill, S., Slyadnev, M. and Stroganov, A. (2018) Identification and Quantitation of Cashmere (Pashmina) Fiber and Wool Using Novel Microchip Based Real-Time PCR Technology. Journal of Textile Science and Technology, 4, 141-150.

https://doi.org/10.4236/jtst.2018.44010

Received: October 14, 2018

Accepted: November 18, 2018

Published: November 22, 2018

Copyright () 2018 by authors and Scientific Research Publishing Inc. This work is licensed under the Creative Commons Attribution International License (CC BY 4.0).

http://creativecommons.org/licenses/by/4.0/

(c) (i) Open Access

\begin{abstract}
The textile industrial chain all over the world is facing a challenge of differentiating cashmere fiber from mixture of wool and other fibers in case cashmere stocks are adulterated with wool or other fibers. For identification of cashmere in such mixtures, the development of microchip based real-time PCR technology offers a very sensitive, specific, and accurate solution. The technology has been validated with cashmere and wool samples procured from distant farms, and from cashmere goats and sheep of different age and sex. Model samples with incremental raw cashmere or wool content were tested. The experimentally determined content was found to be comparable to the weighed content of the respective fibers in the samples. This technology may prove a cost cutter since it needs only $1.2 \mu \mathrm{l}$ of the PCR reagent mix. It is substantially faster than traditional real-time PCR systems for being carried as miniature reaction volume in metal microchip. These features allow faster thermal equilibrium and thermal uniformity over the entire array of microreactors. For routine tests or in commercial set up, the microchips are available as ready-to-run with lyophilized reagents in its microreactors to which only $1 \mu \mathrm{l}$ of the 10 -fold diluted isolated DNA sample is added. The lyophilized microchips offer user-friendly handling in testing laboratories and help minimize human error.
\end{abstract}

\section{Keywords}

Microchip, Real-Time PCR, Identification, Quantitation, Cashmere, Wool

\section{Introduction}

Cashmere, also known as Pashmina, is a luxury fiber derived from cashmere goat Capra hircus and other goats. Although cashmere and wool are two protein 
fibers with analogous geometrical attributes, distinct physical properties of cashmere make it much more expensive than wool. Therefore, in the textile industrial chain, cashmere is often adulterated with sheep wool for material gains. Quantitative analysis of cashmere-wool blends remains difficult by traditional analysis methods. Scanning electron microscopy (SEM) is the only established method for identification (ID) of fibers according to an international standard. However, it is expensive, time consuming, and fails if cashmere is adulterated with descaled or stretched wool or from new breeds of cashmere goats [1] [2] [3] [4].

In order to address the limitations of the SEM in differentiating these fibers in the blends, some well-investigated methods based on protein fingerprinting were developed. Protein analysis of animal hair with mass spectrometry (MALDI-ToF-MS) was considered a highly sensitive and versatile tool [5] [6]. However, these methods are costly and tend to fail when applied in practice.

DNA-analysis of mitochondrion DNA (mtDNA) extracted from animal hair or fibers through DNA amplification techniques (end point PCR or real-time PCR), is able to detect even a minute amount of these fibers [7]-[13]. With this technique, not only cashmere and wool would be distinguished but also their content in a cashmere-wool mixture can be quantified. Additionally, DNA exhibits a relatively strong capacity to resist physical and chemical treatments; this technology may work on dyed fibers as well. The value of cycle threshold $(\mathrm{Ct})$ is used to determine the quantity of the cashmere fiber which is inversely proportional to original mtDNA quantity and thus can be used to quantify amount of mtDNA in unknown samples. But the limited amount of DNA extracted from these fibers and the expensive cost of the reagents prohibitively limits the application.

In view of these limitations, the Microchip based Real-time PCR Technology has been developed to solve the problems encountered in cashmere identification and quantitation. This technology has been widely applied in diverse applications [14] [15]. For cashmere identification and quantitation, this method involves TaqMan polymerase chain reaction (PCR) primers and probes that bind specifically to goat and sheep mitochondrial $12 \mathrm{~S}$ ribosomal RNA gene (mtDNA). The real-time PCR reactions are performed as miniaturized reactions of $1.2 \mu \mathrm{l}$ in microreactors of the microchip. Therefore, this technology offers the following advantages over the traditional real-time PCR for cashmere-wool identification and quantitation:

1) Multiplex real-time PCR are not applicable to this application since goat and sheep are very close in genetic sequences leading to cross reaction of the primers/probes of these animals in the multiplex. To avoid this, the PCR needs to be run in separate tubes of the traditional real-time PCR thereby increasing the cost of analysis. The miniaturization offered by the microchip makes the analysis in separate microreactors of the chip cost effective.

2) Microchip based real-time PCR technology saves time due to fast ramping (18 minutes for 45 cycles) in comparison to other systems. The uniformity of temperature distribution achieved in microreactors made of aluminum (strong 
thermal conductor) in contrast to conventional plastic tubes, offers accuracy. Due to these features, the technology turns out to be highly sensitive and specific compared to the existing technologies as it can report even single copy of the target DNA present in the microreactor.

3) The tube based real-time PCR suffers from the limitation of extracting enough DNA from cashmere fibers [12]. But the microchip based real-time PCR requires a miniaturized amount of DNA ( $1 \mu \mathrm{l}$ of 10 -fold diluted DNA extracted from the animal fibers) and thus extracted DNA can suffice for the tests.

4) A 2-plex test system can be applied to the microchips where animal-specific and Internal Control (IC) results are generated from the same sample to confirm the absence of inhibitors in the sample. This feature also increases the confidence in the test and adds to the cost effectiveness of the microchips.

5) The technology also offers ready-to-use microchips with lyophilized reagents that add to user-friendliness and help minimize human error since the test-lab technician just needs to add $1 \mu \mathrm{l}$ of the 10-fold diluted isolated DNA sample to the microreactors of the chip. Color coded microreactors can also be included to minimize human error.

6) Transportation costs of the ready-to-use microchips are low since the reagents are lyophilized for transportation at ambient temperature free from cold chain.

7) This technology is applicable to identification of other animal-derived fibers as well.

\section{Materials \& Methods}

\subsection{DNA Extraction}

The genomic DNA (gDNA), and mitochondrial DNA (mtDNA) was extracted from $10 \mathrm{mg}$ of cashmere fiber, sheep wool, and hair of other animals. Each sample was digested with proteinase $\mathrm{K}$ at $56^{\circ} \mathrm{C}$ for 4 hours, and subsequently digested with lysis buffer. It was followed by washes with wash buffer, and eventually eluted in $50 \mu \mathrm{l}$ of the elution buffer using Tissue and Hair Extraction Kit and DNA IQ Kit (https://www.promega.com).

\subsection{PCR}

The qPCR mix was formulated with Primetime Gene Expression Master Mix obtained from IDT Inc (https://www.idtdna.com). Primers and probes for detection of goat, sheep, bovine, or common DNA sequences were ordered for synthesis from IDT Inc. To avoid any cross reaction among the primers of goat and sheep, 2-plex PCR was not set up and FAM dye was used for probe labelling. Real-time PCR was run at thermal cycling conditions for 45 cycles as $94^{\circ} \mathrm{C}$ denaturation and $60^{\circ} \mathrm{C}$ extension process.

\subsection{Loading of Master Mix in Microchips}

The qPCR was performed on Microchip qPCR Analyzer, AriaDNA ${ }^{\mathrm{TM}}$ 
(http://www.lumexinstruments.com). The miniature volume $(1.2 \mu \mathrm{l})$ of the reagent mix including the test sample were loaded into each of the microreactors of $6 \times 5$ format microchip using electronic pipettor. The microchip was filled with $620 \mu \mathrm{l}$ silicone oil meant to prevent evaporation during thermocycling.

\section{Results and Discussion}

With the objective of developing a microchip based real-time PCR technology for discrimination of cashmere fibers from wool, it was essential to design specific primer and probe sets to identify signature sequences in mtDNA extracted from these fibers. It was also important to ensure the specificity and sensitivity of these primer-probe sets to setup the test kits for this purpose. Upon testing, the primers and probes for the goat, sheep, and common kits were observed to be responsive and discriminative at a miniaturized volume of $1.2 \mu \mathrm{l}$ in the microchip. The thermal cycling conditions (denaturation: $94^{\circ} \mathrm{C}$, extension and reporting $60^{\circ} \mathrm{C}$ ) offered by the AriaDNA ${ }^{\mathrm{TM}}$ analyzer were observed to be uniform and stable over the entire microchip.

The test kit for goat mtDNA displayed high specificity for the mtDNA extracted from pure cashmere (goat) fibers and generated an early Ct of 16.9 but generated mild cross-reactivity (Ct 28.5) against the sheep test kit as seen in Figure 1(a). On the other hand, the test kit for sheep displayed high specificity (Ct 18.2) for the mtDNA extracted from pure sheep wool displaying mild cross reactivity with cashmere goat kit (Ct 28.3). This data is in agreement with published reports on cashmere and wool fibers performed with traditional real-time PCR [13]. The common test kit meant to detect common DNA sequences of goat and sheep in extracted mtDNA, had Ct of 17.3 and 17.1 for mtDNA extracted from pure cashmere and pure wool fibers, respectively. The early Ct value for both the pure cashmere fiber and pure wool suggested that the test kits would be promising in discrimination of these fibers in the test of their purity and adulteration (Figure 1(b)).

The common, cashmere, and wool test kits were also tested against various meat/fiber/hair of other animals to determine the specificity of these test kits for the base sequences in respective mtDNA extracted from these materials. The data obtained with these test kits indicated high specificity for the respective targets eliminating chance of cross reactivity among the kits (Table 1). Similarly, the stability of the test kit among variable cashmere samples, and wool samples was also tested. The stability of the test kits was reflected by consistent $\mathrm{Ct}$ values and compact replicates $(n=5)$ where cashmere fiber or wool were tested from different sex, color, and age of cashmere goats (Table 2 and Table 3 ).

The results achieved on specificity and sensitivity of the test kits suggested that quantitative determination of cashmere and wool in the blends could be employed for the determination of respective fiber content in the blend. Therefore, a standard curve for incremental quantity of cashmere was generated and accordingly, the percent quantitative determination of cashmere fiber content in 
Table 1. Common, cashmere, and wool test kits vs meat/fiber/hair of other animals to determine the specificity for the sequences in respective mtDNA.

\begin{tabular}{ccccccccccc}
\hline & \multicolumn{3}{c}{$\begin{array}{c}\text { Common } \\
\text { Samples }\end{array}$} & \multicolumn{2}{c}{ Primer-Probe } & \multicolumn{3}{c}{$\begin{array}{c}\text { Goat } \\
\text { Primer-Probe }\end{array}$} & \multicolumn{3}{c}{$\begin{array}{c}\text { Sheep } \\
\text { Primer-Probe }\end{array}$} \\
\cline { 2 - 11 } & Ct & CV\% & Signal & Ct & CV\% & Signal & Ct & CV\% & Signal \\
\hline Cow meat & $-\mathrm{ve}$ & & & $-\mathrm{ve}$ & & & $-\mathrm{ve}$ & & \\
Pork meat & $-\mathrm{ve}$ & & & $-\mathrm{ve}$ & & & $-\mathrm{ve}$ & & \\
Goat meat & 18.6 & 0.2 & 6800 & 18.5 & 0.2 & 6000 & 33.3 & 9.4 & 8000 \\
Sheep meat & 15.2 & 0.6 & 7500 & 27.2 & 0.9 & 5000 & 15.3 & 0.7 & 5500 \\
Cashmere goat fiber & 17.3 & 0.4 & 2000 & 16.9 & 0.8 & 6900 & 28.5 & 0.8 & 7900 \\
Merino sheep wool & 19.3 & 0.9 & 2000 & 31.7 & 2.1 & 100 & 20.9 & 0.4 & 5400 \\
Angora rabbit wool & 28.0 & 1.0 & 2000 & 29.1 & 1.1 & 7000 & 30.5 & 1.3 & 8500 \\
Camel hair & $-\mathrm{ve}$ & & & $-\mathrm{ve}$ & & & $-\mathrm{ve}$ & & \\
Alpaca hair & 28.7 & 1.1 & 2000 & 38.9 & 3.8 & 100 & 28.3 & 1.7 & 6000 \\
Husky Dog hair & 31.5 & 3.4 & 2800 & 31.8 & 0.7 & 4500 & 32.5 & 1.0 & 4500 \\
Buffalo hair & 20.9 & 0.6 & 4800 & 26.2 & 0.8 & 4800 & 30.3 & 4.3 & 5700 \\
Polyester fibers & $-\mathrm{Ve}$ & & & $-\mathrm{Ne}$ & & & $-\mathrm{Ve}$ & & \\
\hline
\end{tabular}

Table 2. Cashmere fiber from cashmere goats of different sex, color, and age.

\begin{tabular}{ccccccccccc}
\hline $\begin{array}{c}\text { Cashmere fiber sample } \\
\text { (sex, color, and age) }\end{array}$ & \multicolumn{3}{c}{ Cashmere P-P } & \multicolumn{3}{c}{ Common P-P } & \multicolumn{3}{c}{ Sheep P-P } \\
\cline { 2 - 9 } & Ct & CV\% & Signal & Ct & CV\% & Signal & Ct & CV\% Signal \\
\hline S1 CBG Thomas 2017, M Dark Brown & 15.9 & 0.9 & 5600 & 15.8 & 1.4 & 5600 & 28.9 & 6.9 & 6200 \\
S2 CBG Hannah 2017, F Dark Brown & 16.2 & 1.2 & 6500 & 16.4 & 0.2 & 5200 & 28.8 & 3.7 & 5200 \\
S3 BLF Flora 2016, F Light Brown & 17.0 & 0.5 & 5400 & 16.9 & 1.6 & 6000 & 29.0 & 4.6 & 4000 \\
S4 CBG Clover 2016, M Dark Brown & 15.5 & 0.0 & 4500 & 16.0 & 0.1 & 5100 & 27.8 & 2.0 & 4200 \\
S5 CBG Fasthing 2018, M White & 16.5 & 1.3 & 6000 & 16.5 & 1.4 & 6000 & 28.5 & 2.5 & 4800 \\
S6 STG Cecily 2018, F White & 16.6 & 0.8 & 4600 & 16.9 & 1.3 & 5700 & 29.7 & 2.2 & 4500 \\
S7 CBG Martha 2018, F Light Brown & 17.2 & 0.7 & 4200 & 16.8 & 1.7 & 6400 & 30.1 & 3.7 & 4700 \\
S8 STC Peace 2018, M White & 16.2 & 1.0 & 5500 & 16.5 & 1.0 & 5100 & 31.0 & 4.5 & 4700 \\
S9 CBG Leo 2018, M Light Brown & 16.1 & 1.4 & 6000 & 16.1 & 0.6 & 6000 & 30.3 & 3.5 & 5000 \\
S10 CBG Lollie 2018, F Dark Brown & 16.8 & 2.5 & 5200 & 16.7 & 2.1 & 5700 & 31.2 & 2.9 & 4100 \\
S11 CBG Dollie 2018, F Whitish Brown & 16.8 & 0.9 & 5400 & 17.1 & 0.4 & 5400 & 33.8 & 2.3 & 2900 \\
S12 CBG Ray 2018, M Light Brown & 15.8 & 2.6 & 4500 & 15.8 & 2.7 & 6800 & 28.7 & 3.2 & 4700 \\
\hline
\end{tabular}

Table 3. Sheep wool from sheep of different sex, color, and age.

\begin{tabular}{ccccccccccc}
\hline $\begin{array}{c}\text { Sheep wool sample } \\
\text { (sex, color, and age) }\end{array}$ & \multicolumn{3}{c}{ Cashmere P-P } & \multicolumn{3}{c}{ Common P-P } & \multicolumn{3}{c}{ Sheep P-P } \\
\cline { 2 - 9 } & Ct & CV\% & Signal & Ct & CV\% & Signal & Ct & CV\% Signal \\
\hline S1 BFF, Koki, 2016, Fe Black & 29.3 & 2.8 & 4500 & 17.3 & 2.3 & 4800 & 18.3 & 0.3 & 4800 \\
S2 BFF, Dolly 2017, F Black & 28.7 & 2.1 & 4500 & 16.3 & 0.8 & 6400 & 15.5 & 0.9 & 5600 \\
S3 BFF, Kaunu 2016, M Light Brown & 28.3 & 3.5 & 6700 & 19.9 & 3.4 & 5900 & 21.7 & 3.4 & 7100 \\
S4 BFF, Doba 2017, M White & 26.3 & 2.1 & 4700 & 16.8 & 0.5 & 5900 & 17.8 & 0.7 & 5500 \\
S5 BFF, Fiona 2018, F White & 28.0 & 2.17 & 5400 & 17.8 & 0.9 & 5300 & 18.4 & 1.3 & 5900 \\
S6 BFF, Lamb Tuli 2018, M Black & 30.3 & 1.3 & 4400 & 15.5 & 0.6 & 6000 & 16.3 & 0.5 & 6800 \\
\hline
\end{tabular}




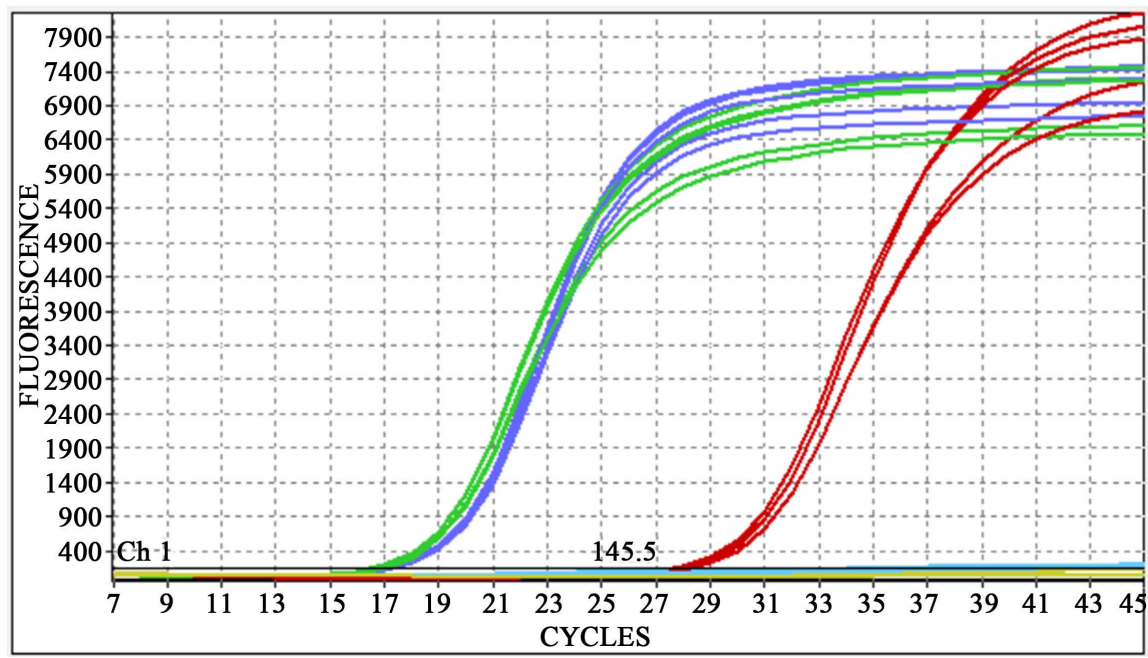

(a)

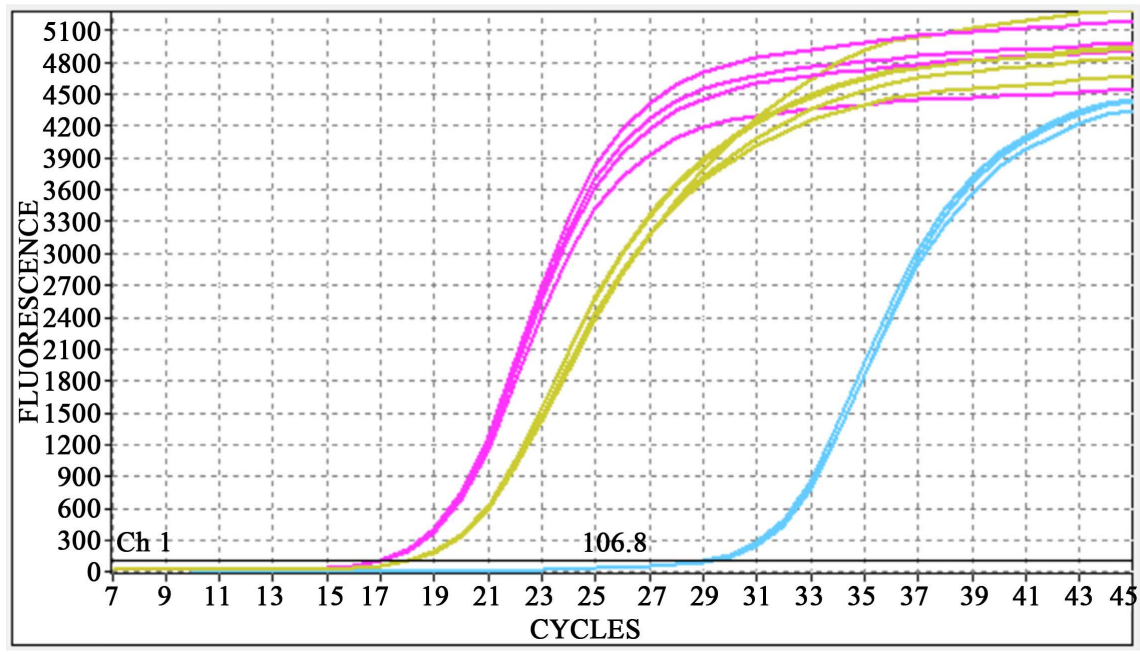

(b)

Figure 1. (a) Specificity of the goat test kit for mtDNA extracted from cashmere fibers. Cashmere goat test kit Ct of 16.9 (0.8\% CV, green curve), and sheep test kit (Ct 28.5, 0.8\% $\mathrm{CV}$, red curve); (b) Specificity of sheep test kit for mtDNA extracted from sheep wool. Sheep test kit Ct of 18.2 (0.3\% CV, brown curve) and cashmere goat test kit (Ct 28.3, 1.1\% CV, sky blue curve).

unknown samples was determined (Figure 2(a)). An amplification of the sets of 5 model samples (cashmere $7.5 \mathrm{mg}+$ wool $2.5 \mathrm{mg}$; and cashmere $9.0+$ wool 1.0 $\mathrm{mg}$ ) was also performed. These amplifications of the cashmere goat and sheep mtDNA indicated compactness among replicates $(n=3)$. The data generated from these 5 model test samples also displayed high PCR efficiency (99.1\%) and CV repeatability of $<5 \%$ (Figure $2(\mathrm{~b})$ ). A precision of $1.84 \%$ and $2.0 \%$ was achieved for cashmere determination in the model sample tests of $75 \%$, and $90 \%$ cashmere content presented in Table 4.

For commercial applications, a layout was designed for the test microchips (Figure 3). This microchip layout allows both qualitative and quantitative determinations of natural fibers of cashmere and wool in the unknown samples. 


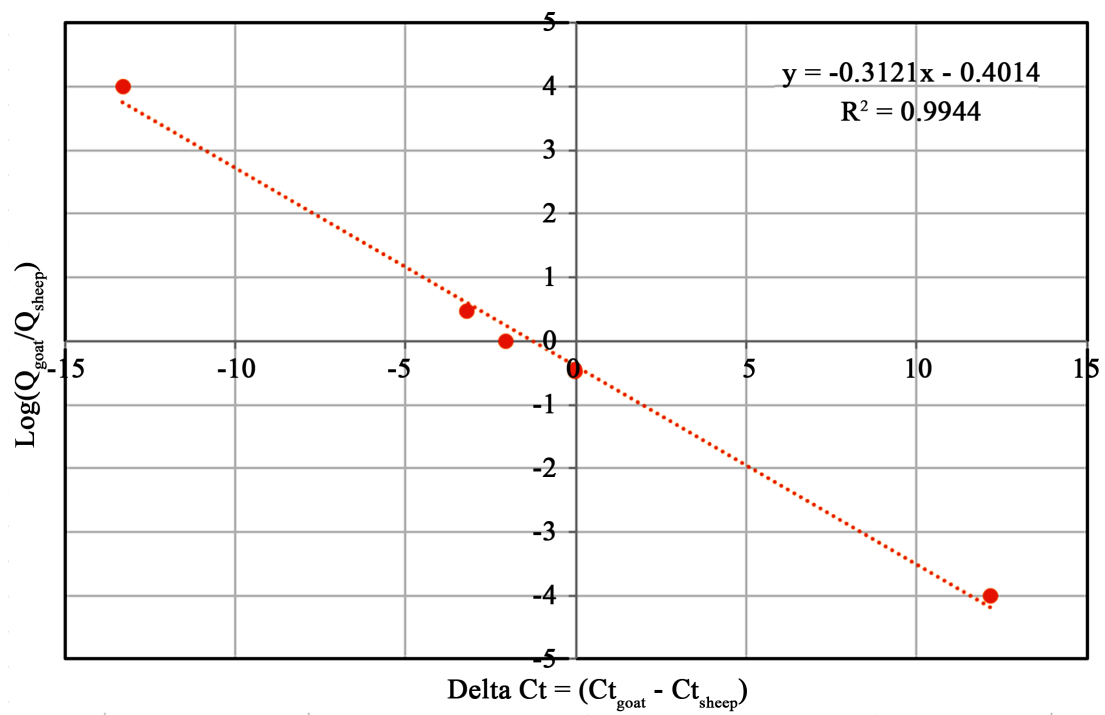

(a)

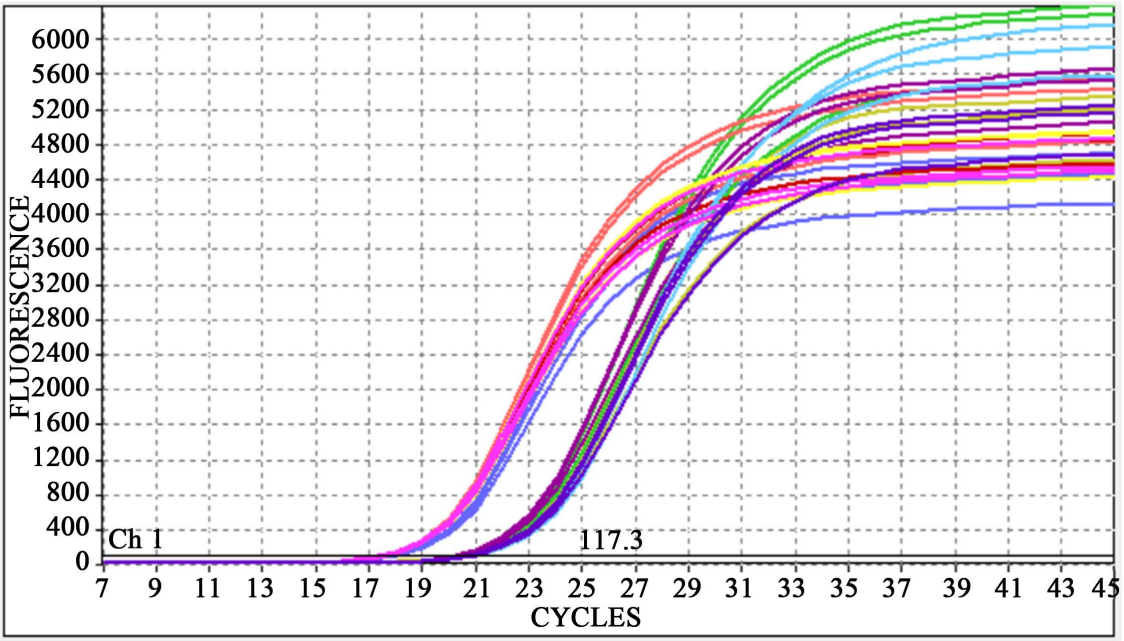

(b)

Figure 2. (a) Standard curve for determination of percentage of cashmere fiber; (b) Amplification curves of a set of 5 model samples (cashmere $7.5 \mathrm{mg}$ + wool $2.5 \mathrm{mg}$ ) displaying goat test kit: blue, red, light purple, pink, and yellow. Sheep test kit: green, sky blue, brown, dark purple, and navy $(\mathrm{n}=3)$.

Table 4. Cashmere determination in the model sample tests.

\begin{tabular}{cccccc}
\hline $\begin{array}{c}\text { Unknown } \\
\text { model } \\
\text { sample }\end{array}$ & $\begin{array}{c}\text { Input } \\
\text { cashmere } \\
\text { content (\%) }\end{array}$ & $\begin{array}{c}\text { Experimental } \\
\text { cashmere } \\
\text { content (\%) }\end{array}$ & $\begin{array}{c}\text { Unknown } \\
\text { model } \\
\text { sample }\end{array}$ & $\begin{array}{c}\text { Input } \\
\text { cashmere } \\
\text { content (\%) }\end{array}$ & $\begin{array}{c}\text { Experimental } \\
\text { cashmere } \\
\text { content (\%) }\end{array}$ \\
\hline Sample 1 & 75 & 74.9 & Sample 6 & 90 & 90.7 \\
Sample 2 & 75 & 69.6 & Sample 7 & 90 & 86.9 \\
Sample 3 & 75 & 80.3 & Sample 8 & 90 & 89.7 \\
Sample 4 & 75 & 78.7 & Sample 9 & 90 & 86.7 \\
Sample 5 & 75 & 74.1 & Sample 10 & 90 & 89.6 \\
Mean & & 75.5 & Mean & 88.7 \\
SD (\%) & 4.2 & SD (\%) & 1.8 \\
CV\% & 5.6 & CV (\%) & 2.0 \\
\hline
\end{tabular}




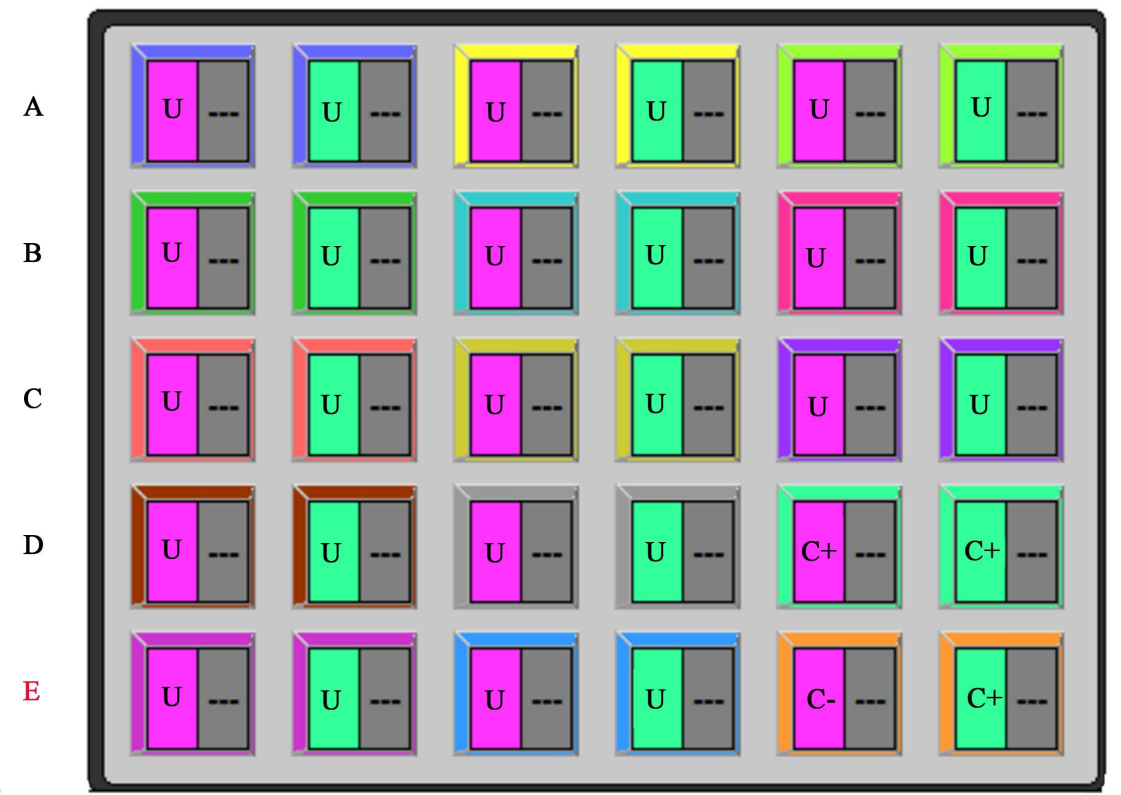

Figure 3. Microchip layout map for qualitative and quantitative cashmere and wool determination in natural fibers.

The proposed template accommodates 6 unknown samples $(\mathrm{n}=2)$ or 13 unknown samples $(\mathrm{n}=1)$ under investigation, 1 negative control $(\mathrm{NC})$, and 1 positive control (PC). The IC can also be added to each sample to ensure confidence in data. The test microchips with this standard layout were also lyophilized to be used as ready-to-run microchips to help minimize the human error by minimized liquid handling and to provide increased throughput in testing the fibers.

\section{Conclusion}

The novel microchip based real-time PCR may prove a valuable technology for identifying and quantifying cashmere and wool content in the fiber mixtures like wool adulteration of cashmere. The data on cashmere samples procured from different regions and from cashmere goats of different age supports the validity of this technology. Being performed as $1.2 \mu \mathrm{l}$ reaction-volume in metal microreactors, the test would be cost effective, highly specific \& sensitive, with minimized human error, and minimal false negative or false positive rates. This method based on testing mtDNA extracted from the cashmere and wool can be used for mass-testing and for this purpose, the ready-to-run microchips may prove user-friendly to the technical lab staff.

\section{Acknowledgements}

The authors are thankful to Dr. A.K. Sharma, Director General; and Dr. Seema Patel, Joint Director, Wool Research Institute, Thane, India for valuable discussion and animal hair \& fiber samples. The supplying of cashmere and wool samples by Mrs. Maggie Constantine Porter, Casa Blanca Goat Farm, Center Sand- 
wich, NH, USA; Mr. Mathew Laity, Brookfield Farm, Maple Ridge, BC, Canada; and Mrs. Barbara Fiorica, Caprette Cashmere Farm, Wilton, CA, USA is also acknowledged. Our thanks are also due to Ms. Brooke Morrison for editing the manuscript.

\section{Conflicts of Interest}

The authors declare no conflicts of interest regarding the publication of this paper.

\section{References}

[1] ISO 17751-1 (2016) Textiles-Quantitative Analysis of Cashmere, Wool, Other Specialty Animal Fibers and Their Blends-Part 1: Light Microscopy Method. https://www.iso.org/obp/ui/\#iso:std:iso:17751:-1:ed-1:v1:en

[2] Coelho, L.S., Modesto, E.C., Carvalho, F.F.R. and de Sousa, S.L.G. (2018) Characterization and Identification of Cashmere in Goats in Northeastern Brazil. Revista Acadêmica: Ciência Animal, 16, 1-7. https://doi.org/10.7213/1981-4178.2018.16007

[3] Zhong, Y., Lu, K., Tian, J. and Zhu, H. (2017) Wool/Cashmere Identification Based on Projection Curves. Textile Research Journal, 87, 1730-1741.

https://doi.org/10.1177/0040517516658516

[4] Zhou, J., Wang, R., Wu, X. and Xu, B. (2017) Fiber-Content Measurement of Wool-Cashmere Blends Using Near-Infrared Spectroscopy. Applied Spectroscopy, 71, 2367-2376. https://doi.org/10.1177/0003702817713480

[5] Izuchi, Y., Tokuhara, M., Takashima, T. and Kuramoto, K. (2013) Peptide Profiling Using Matrix-Assisted Laser Desorption/Ionization-Time-of-Flight Mass Spectrometry for Identification of Animal Fibers. Mass Spectrum (Tokyo), 2, A0023. https://www.ncbi.nlm.nih.gov/pmc/articles/PMC3967007/

[6] Kim, Y., Kim, T. and Choi, H.M. (2013) Qualitative Identification of Cashmere and Yak Fibers by Protein Fingerprint Analysis Using Matrix-Assisted Laser Desorption/Ionization Time-of-Flight Mass Spectrometry. Industrial Engineering Chemistry, 52, 5563-5571. https://doi.org/10.1021/ie4003415

[7] Geng, R.Q., Yuan, C. and Chen, Y.L. (2012) Identification of Goat Cashmere and Sheep Wool by PCR-RFLP Analysis of Mitochondrial 12S rRNA Gene. Mitochondrial DNA, 23, 466-4670. https://doi.org/10.3109/19401736.2012.710206

[8] Ji, W., Bai, L., Ji, M. and Yang, X.A. (2011) Method for Quantifying Mixed Goat Cashmere and Sheep Wool. Forensic Science International, 208, 139-142. https://doi.org/10.1016/j.forsciint.2010.11.017

[9] Sun, M.R., Fei, J., Cai, J.S. and Zhao, J. (2016) Application of DNA Analysis in Quantifying Cashmere and Wool Binary Blend. Key Engineering Materials, 67, 378-384. https://doi.org/10.4028/www.scientific.net/KEM.671.378

[10] Geng, Q.R. (2016) A Duplex Polymerase Chain Reaction Assay for the Identification of Goat Cashmere and Sheep Wool. Journal of Mitochondrial DNA, 27, 1808-1811. https://doi.org/10.3109/19401736.2014.963823

[11] Navolotskii, D.V., Perchik, A.V., Markyanov, I.A., Ganeev, A.A. and Slyadnev, M.N. (2015) Microchip Analytic System for Multiplex Analysis by Real-Time Polymerase Chain Reaction with Reagents Immobilized in Microreactors. Applied Biochemistry and Microbiology, 47, 221-227. https://doi.org/10.1134/S0003683811020141

[12] Kumar, R., Shakyawar, D.B., Pareek, P.K., Raja, A.S., Prince, L.L., Kumar, S. and 
Naqvi. S.M. (2015) Development of PCR-Based Technique for Detection of Purity of Pashmina Fiber from Textile Materials. Applied Biochemistry and Biotechnolgy, 175, 3856-3862. https://doi.org/10.1007/s12010-015-1552-Z

[13] Tang, M., Zhang, W., Zhou, H., Fei, J., Yang, J., Lu, W., Zhang, S., Ye, S. and Wang, X.A. (2014) A Real-Time PCR Method for Quantifying Mixed Cashmere and Wool Based on Hair Mitochondrial DNA. Textile Research Journal, 84, 1612-1621. https://doi.org/10.1177/0040517513494252

[14] Gill, S., Slyadnev, M.N. and Stroganov, A. (2016) Optimization of Paramagnetic Bead Based Nucleic Acid Isolation for High Throughput Potato Pathogen Detection Using Microchip Real-Time PCR. Laboratory Focus, 20, 8-11.

https://laboratoryfocus.ca/optimization-

[15] Nikitin, M.M., Statsyuk, N.V., Frantsuzov, P.A., Dzhavakhiya, V.G. and Golikov, A.G. (2018) Matrix Approach to the Simultaneous Detection of Multiple Potato Pathogens by Real-Time PCR. Journal of Applied Microcbiology, 124, 797-809.

https://doi.org/10.1111/jam.13686 